\title{
Impacto da ultrassonografia doppler no exame clínico andrológico de reprodutores
}

\section{equinos}

\author{
Impact of doppler ultrasound on the clinical and andrological stallions examination
}

Impacto de la ecografía Doppler en la exploración clínica y andrológica de los sementales

\author{
Allan Gledson Ferreira dos Santos \\ ORCID: https://orcid.org/0000-0002-6841-8660 \\ Universidade Federal da Paraíba, Brasil \\ E-mail: allan.santos@ceva.com \\ Hugo Albino Braz \\ ORCID: https://orcid.org/0000-0001-5848-0076 \\ Universidade Federal da Paraíba, Brasil \\ E-mail: hugalbino@gmail.com \\ Robespierre Augusto Joaquim Araújo Silva \\ ORCID: https://orcid.org/0000-0002-5521-8571 \\ Singamia Reprodução Pet, Brasil \\ E-mail: robespierreaugusto@yahoo.com.br \\ Maria Madalena Pessoa Guerra \\ ORCID: https://orcid.org/0000-0002-5373-4062 \\ Universidade Federal Rural de Pernambuco, Brasil \\ E-mail: mmpguerra@gmail.com \\ Marlon de Vasconcelos Azevedo \\ ORCID: https://orcid.org/0000-0001-7751-7815 \\ Universidade Federal Rural de Pernambuco, Brasil \\ E-mail: marlon.a.vet@gmail.com.br \\ Sildivane Valcácia Silva \\ ORCID: https://orcid.org/0000-0003-1488-8074 \\ Universidade Federal da Paraíba, Brasil \\ E-mail: sildivane@cbiotec.ufpb.br
}

\begin{abstract}
Resumo
Objetivou-se avaliar a associação da ultrassonografia Doppler com o exame clínico andrológico para identificação da qualidade reprodutiva de equinos no Brejo paraibano. Foram utilizados 11 equinos da raça Quarto de milha, com idade entre três a 27 anos. Os ejaculados foram colhidos pelo método de vagina artificial e as amostras seminais submetidas à avaliação a campo (motilidade subjetiva, integridade celular) e em laboratório (motilidade objetiva, integridade celular). Após colheita seminal, os animais foram submetidos à avaliação do fluxo sanguíneo testicular por ultrassonografia doppler. Diferenças $(p<0,05)$ foram observadas entre os parâmetros de motilidade subjetiva, avaliada a campo, e motilidade objetiva, no laboratório. A integridade da membrana plasmática espermática foi semelhante nos testes a campo e em laboratório. Nos parâmetros ultrassonográficos, observou-se que o Índice de Pulsatilidade tem correlação positiva com amplitude de deslocamento lateral de cabeça (ALH) do espermatozoide. Por fim, com o aumento da idade dos reprodutores, observou-se redução da motilidade e menor aporte sanguíneo na região testicular, resultando em menor potencial de fertilidade. Conclui-se que o exame clínico andrológico, quando isolado, não determina a capacidade fértil de garanhões; a ultrassonografia associada ao método doppler identifica animais subférteis e pode ser uma ferramenta auxiliar na seleção de reprodutores equinos.
\end{abstract}

Palavras-chave: Fertilidade; Índice de resistência; Membranas espermáticas.

\begin{abstract}
This study aimed to evaluate the association of Doppler ultrasound with clinical andrological examination to identify the reproductive quality of stallions in paraiban swamp region. Eleven horses were used, aged between three and 27 years. The ejaculates were collected by the artificial vagina method and the seminal samples were submitted to field evaluation (subjective motility, cellular integrity) and in the laboratory (objective motility, cellular integrity). After seminal collection, the animals were submitted to testicular blood flow evaluation with Doppler ultrasound. Differences $(\mathrm{P}<0.05)$ were observed between the parameters of subjective motility, field-evaluated, and objective motility, in the laboratory. The integrity of the sperm plasma membrane was similar in field and laboratory tests. In the ultrasound parameters, it was observed that the Pulsatility Index has a positive correlation with lateral head displacement amplitude (ALH) of the spermatozoa. Finally, when evaluating age parameter, elderly animals presented reduced motility and lower blood supply in the testicular region, resulting in low fertility. It is concluded
\end{abstract}


that the clinical andrological examination, when isolated, does not determine the fertile capacity of stallions; dopplerassociated ultrasound identifies subfertile animals and can be an auxiliary tool in the stallions selection.

Keywords: Fertility; Resistive index; Sperm membranes.

\begin{abstract}
Resumen
Este estudio tuvo como objetivo evaluar la asociación de la eco-grafía Doppler con el examen clínico andrológico para identificar la calidad reproductiva de los sementales en la región del marisma de Paraíba. Se utilizaron once caballos, con edades comprendidas entre los tres y los 27 años. Los eyaculados fueron recolectados por el método de vagina artificial y las muestras seminales fueron sometidas a evaluación de campo (motilidad subjetiva, integridad celular) y en el laboratorio (motilidad objetiva, integridad celular). Después de la recolección seminal, los animales se sometieron a evaluación del flujo sanguíneo testicular con ultrasonido Doppler. Se observaron diferencias $(\mathrm{P}<0.05)$ entre los parámetros de motilidad subjetiva, evaluados en campo y motilidad objetiva, en el laboratorio. La integridad de la membrana plasmática de los espermatozoides fue similar en las pruebas de campo y de laboratorio. En los parámetros eco-gráficos, se observó que el Índice de Pulsatilidad tiene una correlación positiva con la amplitud de desplazamiento lateral de la cabeza (ALH) de los espermatozoides. Finalmente, al evaluar el parámetro de edad, los animales de edad avanzada presentaron menor motilidad y menor riego sanguíneo en la región testicular, resultando en baja fertilidad. Se concluye que el examen clínico andrológico, cuando se aísla, no determina la capacidad fértil de los sementales; La eco-grafía asociada a Doppler identifica animales subfertiles y puede ser una herramienta auxiliar en la selección de sementales.
\end{abstract}

Palabras clave: Fertilidad; Índice de resistencia; Membranas de esperma.

\title{
1. Introdução
}

O complexo do agronegócio equino no Brasil é uma atividade que movimenta, por ano, $\mathrm{R} \$ 16,15$ bilhões e gera 612 mil empregos diretos, sendo responsável por desenvolver cerca de três milhões de postos de trabalho de forma indireta (Obino Filho, 2016). Neste contexto, ressalta-se a importância do cavalo no aspecto social, nas atividades de trabalho, esportes e lazer e, ainda, na equoterapia (Pereira et al., 2020). A região Nordeste, por sua vez, é importante impulsionadora da equinocultura devido à vaquejada, esporte que provém da cultura sertaneja nordestina, como grande manifestação popular e grande importância econômica para a região (Linhares \& Sousa, 2020).

A importância econômica de um reprodutor equino é fundamental dentro da equinocultura brasileira, pois muitos animais de alto valor zootécnico são exigidos durante repetidas estações reprodutivas pelo alto valor de suas coberturas. Portanto, falhas na fertilidade destes garanhões acarretam em prejuízos econômicos consideráveis (Hammes et al., 1996).

A espécie equina foi considerada por muito tempo como a de menor fertilidade entre as espécies domésticas, o que foi atribuído a características de seleção e problemas relacionados ao manejo reprodutivo (Ginther, 1992). Desta forma, o exame clínico andrológico passa a ser uma ferramenta de grande importância na comercialização de garanhões e de ejaculados, já que diferentemente das outras espécies domésticas, estes animais não são selecionados pelo seu desempenho reprodutivo, e sim pelo seu pedigree, desempenho esportivo e outras características, como a conformação (Smith et al., 2006).

Nesse aspecto é válido ressaltar a relevância do garanhão na reprodução equina, sendo responsável por produzir grande número de produtos por ano, uma vez que através de um único ejaculado pode fecundar várias éguas quando utilizadas biotécnicas reprodutivas. O exame do potencial de fertilidade de um garanhão inclui todas as etapas do exame clínico andrológico, e é indicado antes de iniciar a estação de monta, exposição, diagnósticos de sub ou infertilidade, ocorrência da puberdade, criopreservação de sêmen e comercialização (Papa et al., 2007).

O exame andrológico permite uma avaliação pontual do ejaculado equino. Ao identificar patologias espermáticas ou diminuição do número de éguas prenhes, exames complementares como a avaliação ultrassonográfica do aparelho reprodutor equino podem fundamentar ou descartar a saúde reprodutiva do garanhão. Sabe-se que alterações significativas no fluxo sanguíneo podem influenciar negativamente a espermatogênese, porém, alterações discretas podem não ser evidenciadas no exame clínico andrológico e podem ser responsáveis pela redução na fertilidade do macho equino (Cunha et al., 2015).

Baseado na importância do equino no agronegócio e a possível inserção de um método de avaliação complementar 
para a rotina clínica andrológica, este trabalho teve por avaliar a relação entre exame andrológico, achados ultrassonográficos e a fertilidade em garanhões no estado da Paraíba.

\section{Metodologia}

O presente trabalho foi desenvolvido na região de Campina Grande/PB ( $7^{\circ} 15^{\text {ce }} 18^{\text {" }}$ de latitude sul e $35^{\circ} 55^{\text {ec }} 28^{\text {" }}$ de longitude oeste), no período entre outubro de 2016 a outubro de 2017. As condições climáticas locais apresentam temperaturas mais moderadas, considerado tropical com estação seca, com chuvas concentradas no outono e inverno, principalmente entre os meses de abril e julho, sendo este último o mês de maior precipitação, com média de 154 milímetros (AESA, 2017).

Os procedimentos realizados nos animais foram aprovados pelo Comitê de Ética no Uso de Animais (CEUA) da Universidade Federal da Paraíba (Protocolo No 124/2016 CEUA/UFPB). Onze equinos da raça quarto de milha foram utilizados, com idade entre três a 27 anos (cinco animais com idade entre três a cinco anos; seis animais a partir de seis anos de idade), e prévio histórico de fertilidade (fêmeas com prenhez confirmada após cópula ou inseminação artificial ou potros nascidos). Os animais eram alimentados com concentrado comercial (6 kg/animal/dia), feno tífton e água ad libitum, mantidos nas fazendas de criação.

Foram realizadas duas colheitas de sêmen por animal, por semana, durante três semanas, totalizando seis amostras por animal. Os ejaculados foram colhidos pelo método de vagina artificial modelo Hannover, com temperatura média de $42{ }^{\circ} \mathrm{C}$. A vagina foi revestida por uma camisinha sanitária e copo coletor composto por filtro de nylon para remoção da fração gel do ejaculado. Utilizou-se para a colheita um manequim natural, uma égua em estro. Após a colheita do sêmen, o ejaculado foi mantido fora da luz, sobre placa aquecedora $\left(37^{\circ} \mathrm{C}\right)$ durante a avaliação. $\mathrm{O}$ sêmen fresco foi avaliado quanto ao volume e aspecto no próprio copo coletor graduado.

O sêmen in natura foi avaliado imediatamente após a colheita (CBRA, 2013) quanto à motilidade total subjetiva (0$100 \%$ ), concentração (x 106/mL), integridade (dupla coloração com os corantes eosina-nigrosina) e funcionalidade de membrana espermática (teste hiposmótico) segundo técnica descrita por Snoeck et al. (2014).

$\mathrm{Na}$ sequência, as amostras foram diluídas em 1:1 com diluente comercial (BotuSêmen®, Botupharma, Brasil), envasadas (BotuIA, Botupharma, Brasil) e acondicionadas em caixa de transporte isotérmica (BotuFLEX®, Botupharma, Brasil), à $15{ }^{\circ} \mathrm{C}$ (Coelho \& Dias, 2021). As amostras, então, foram transportadas para o Laboratório de Andrologia (ANDROLAB), com percurso médio de três horas, para avaliação objetiva da motilidade e da integridade de membrana plasmática e acrossomal.

A avaliação da cinética espermática foi realizada através do sistema computadorizado para análise de espermatozoide (CASA, SCA; Microptics, SL, Versão 5.1, Barcelona, Espanha). Uma alíquota (5 $\mu \mathrm{L})$ de amostra foi colocada sobre lâmina e lamínula $\left(18 \times 18 \mathrm{~mm}\right.$ ); ambas previamente aquecidas a $37^{\circ} \mathrm{C}$ e avaliada por microscopia de contraste de fase (Eclipse 50i, Nikon, Japão). As imagens das células espermáticas foram capturadas usando câmera de vídeo (Basler Vision Technologie A312FC, Ahrensburg, Alemanha), ampliação de 100x. Para cada amostra, foram gravados cinco campos aleatórios, não consecutivos, com observação mínima de 500 espermatozoides por captação. As seguintes variáveis foram avaliadas: motilidade total (MT; \%), motilidade progressiva (MP; \%), velocidade linear progressiva (VSL; $\mu \mathrm{m} / \mathrm{s}$ ), velocidade curvilínea (VCL; $\mu \mathrm{m} / \mathrm{s}$ ), velocidade média do percurso (VAP; $\mu \mathrm{m} / \mathrm{s}$ ), Frequência do batimento flagelar cruzado (BCF; Hz), amplitude do deslocamento lateral de cabeça (ALH; $\mu \mathrm{m}$ ), retilinearilidade (STR; \%) e linearidade (LIN; \%). Os parâmetros do sistema CASA foram medidos com as seguintes configurações: temperatura de $37{ }^{\circ} \mathrm{C}$; ampliação $100 \mathrm{x}$; número de imagens, 25; quadros por segundo, 25; área da cabeça, 20-70 $\mu \mathrm{m}^{2}$; VAP: $10 \mu \mathrm{m} / \mathrm{s}$ lentos; <45 $\mu \mathrm{m} / \mathrm{s}$ médio; $<75 \mu \mathrm{m} / \mathrm{s}$ rápidos; progressividade, $80 \%$ STR; circular, 50\% LIN (Nery et al., 2020).

Para análise da integridade de membrana plasmática e acrossomal (iPAM), $50 \mu \mathrm{L}$ da amostra seminal foi corada com 
5,0 $\mu \mathrm{L}$ de Iodeto de Propídeo (IPD; solução de trabalho: $1 \mathrm{mg} / \mathrm{mL}$ em PBS) e 5,0 $\mu \mathrm{L}$ de Isotiocianato de fluoresceína conjugada à aglutinina do amendoim (FITC-PNA; solução de trabalho: $0,04 \mathrm{mg} / \mathrm{mL}$ em PBS), homogeneizada e incubada a temperatura ambiente por cinco minutos e avaliada por meio de citometria de fluxo (ImageStream x Mark II; EMD Millipore Corp., Seattle, Washington). Um total de 5000 eventos por amostra foi capturado. O tamanho das células e a velocidade do fluxo foi de 7,0 $\mu \mathrm{m}$ e $44 \mathrm{~mm} / \mathrm{seg}$, respectivamente. Células marcadas com FITC-PNA foram coletadas no canal 2 (505-560 $\mathrm{nm})$, e marcadas com o IPD foram recolhidas no canal $5(640-745 \mathrm{~nm})$. O software INSPIRE foi usado, e a análise da imagem foi realizado usando o software de análise de imagem IDEAS 6.0. Os resultados obtidos foram expressos como percentual de células com membrana plasmática intactas (FITC-PNA-/IPD-).

Antes do exame ultrassonográfico, os animais passaram por exame clínico para avaliação da frequência cardíaca, frequência respiratória, temperatura corporal, tempo de perfusão capilar, motilidade intestinal e colheita de sangue para análise hematológica. Para o exame ultrassonográfico, os animais foram contidos em tronco de contenção, mantidos em estação, e submetidos ao exame na região do testículo e cordão espermático. Utilizou-se o aparelho S2V (SonoScape Co., Ltda, China), equipado com transdutor linear e convexo, ambos multifrequenciais (3,5 a $7 \mathrm{MHz}$ ). Todos os exames de ultrassonografia foram realizados pelo mesmo operador, para evitar variação operacional.

Inicialmente, no modo B e na frequência de $7 \mathrm{MHz}$ foi identificada a artéria testicular (Figura 1A) e, após, o modo Power Doppler foi ativado para a avaliação dos parâmetros de fluxo sanguíneo (Figura 1B). Em seguida, o Doppler pulsátil avaliou a velocidade do fluxo sanguíneo vascular (Figuras 2A e 2B), com utilização do ângulo Doppler de $60^{\circ}$. Para avaliação dos parâmetros Doppler foi calculado a Velocidade Sistólica do Pico (VSP), a Velocidade Diastólica Final (VDF) e a Velocidade Máxima do Tempo (VMT); estes valores foram utilizados para o cálculo dos Índices de Resistência (IR: VSPVDF/VSP) e Pulsatilidade (IP: VSP-VDF/VMT).

Figura 1 - Imagem ultrassonográfica do corte transversal da artéria testicular do garanhão em Modo B (A); Posicionamento do Modo Power Doppler no cordão espermático (B)

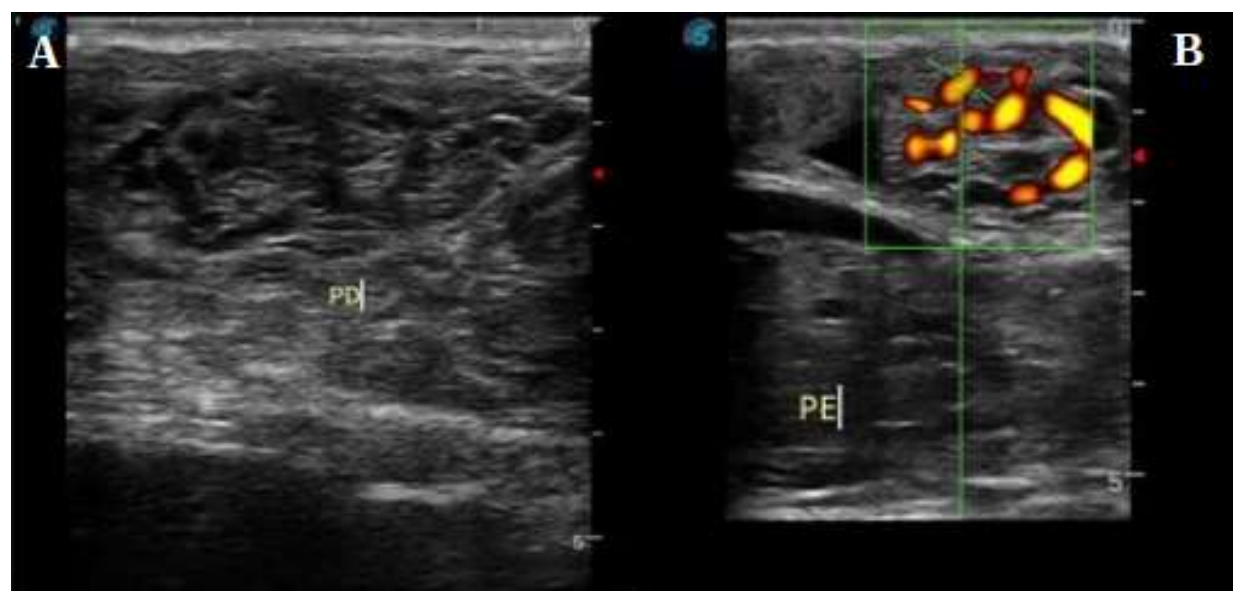

Legenda: Plexo Direito (PD); Plexo Esquerdo (PE). Fonte: Autores. 
Figura 2 - Corte transversal do cordão espermático de um garanhão (A). Modo Power Doppler de cor dos vasos do cordão espermático, exibição do método utilizado para medir um ciclo cardíaco usando Doppler pulsátil (B)

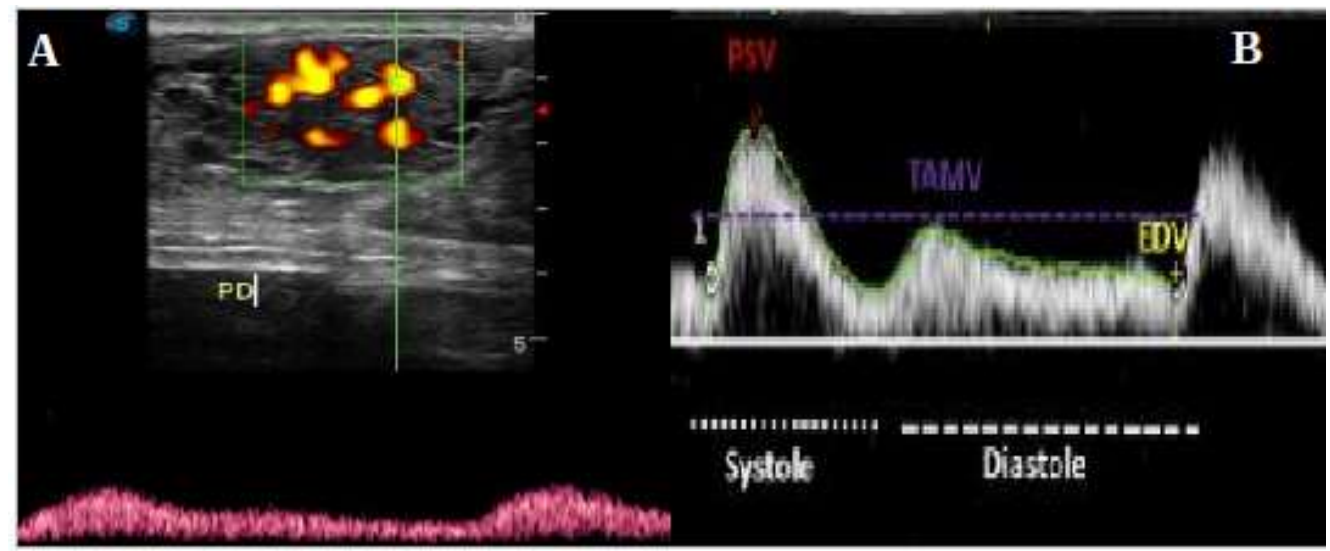

Legenda: Plexo Direito (PD); Velocidade Sistólica do Pico (VSP; PSV em inglês); Velocidade Diastólica Final (VDF; EDV em inglês) e a Velocidade Máxima do Tempo (VMT; TAMV em inglês). Fonte: Autores.

Durante a estação reprodutiva, os garanhões foram submetidos a três colheitas seminais por semana. Cada garanhão foi utilizado para a inseminação de éguas, de acordo com a demanda, ocasionando número variável de éguas inseminadas durante a estação de monta para cada reprodutor. O percentual de fertilidade foi calculado baseado na quantidade de éguas prenhes com uma única inseminação, mensuradas por ultrassonografia, aos 14, 28 e 60 dias pós-inseminação, sendo considerada como prenhez liberada a partir dos 60 dias.

Para análise dos dados, o experimento foi conduzido em delineamento experimental inteiramente casualizado. Os dados foram submetidos à análise de normalidade (Kolmogorov-Sminorv). Os dados não paramétricos foram submetidos à análise de variância $(\mathrm{P}<0,05)$ e as médias foram comparadas pelo teste $\mathrm{F}$, utilizando o software $\mathrm{R}$ versão 3.4 (R Core Team, 2017). Em complemento, realizou-se análise de regressão exponencial e teste de correlação Pearson.

\section{Resultados}

Para os resultados de motilidade, observou-se diferença $(\mathrm{P}<0,05)$ entre os parâmetros de motilidade total subjetiva (67,3\%), avaliada a campo, e motilidade total avaliada no sistema computadorizado de avaliação espermática (44,9\%).

A análise do teste de integridade de membrana plasmática realizada a campo $(43,6 \%$, método dupla coloração com eosina-nigrosina) não apresentou diferença $(\mathrm{P}>0,05)$ quando comparada às análises realizadas no laboratório através das sondas fluoróforas $(42,1 \%)$. Já o teste de funcionalidade de membrana $(56,9 \%$; HOST) apresentou diferença $(\mathrm{P}<0,05)$ quando comparado com a integridade de membrana tanto pelo método de coloração a campo quanto através das sondas fluoróforas.

Não foi observada diferença $(\mathrm{P}>0,05)$ nos parâmetros vitais e na análise hematológica dos animais durante o experimento, independentemente da idade dos animais. Apesar da normalidade destes parâmetros, houve variação $(\mathrm{P}<0,05)$ no percentual da motilidade espermática em relação à idade dos garanhões, ou seja, no intervalo entre as idades de três a cinco anos, os animais apresentaram motilidade espermática $\leq 85,5 \%$ e animais com idade superior a seis anos apresentaram motilidade espermática $\leq 70,3 \%$.

$\mathrm{O}$ índice de resistividade apresentou diferença $(\mathrm{P}<0,05)$ quando comparada à idade dos animais. Os animais, a partir de seis anos de idade, demonstraram um ciclo cardíaco mais curto, aumentando o aporte sanguíneo nos testículos. Ao correlacionar os parâmetros de índice de resistência (IR) e índice de pulsatilidade (IP), e as análises dos parâmetros cinéticos da célula espermática mostraram que o IP tem correlação positiva $(0,65)$ com amplitude de deslocamento lateral da cabeça do espermatozoide (ALH). 
Durante a avaliação da fertilidade dos garanhões na estação reprodutiva (Tabela 1), observou-se que animais jovens (três a cinco anos de idade; valor médio de 82,5\%) apresentaram taxa de fertilidade superior $(\mathrm{P}<0,05)$ aos animais mais velhos (a partir de seis anos; valor médio de 68,6\%), de acordo com a taxa de prenhez obtida no período.

Tabela 1 - Taxa de prenhez obtida com o uso de garanhões quarto de milha de diferentes idades no período de estação reprodutiva no Brejo paraibano

\begin{tabular}{|cc|cc|}
\hline Identificação Animal & $\begin{array}{c}\text { Idade do Garanhão } \\
\text { (anos) }\end{array}$ & $\begin{array}{c}\text { Número de Éguas } \\
\text { Inseminadas }\end{array}$ & Taxa de prenhez (\%) \\
\hline A & 3 & 8 & $(07 / 08 ; 87,5 \%)$ \\
B & 3 & 16 & $(12 / 16 ; 75,0 \%)$ \\
C & 4 & 11 & $(09 / 11 ; 81,8 \%)$ \\
D & 5 & 32 & $(26 / 32 ; 81,2 \%)$ \\
E & 5 & 54 & $(47 / 54 ; 87,0 \%)$ \\
F & 6 & 8 & $(10 / 13 ; 76,9 \%)$ \\
G & 6 & 13 & $(11 / 15 ; 73,3 \%)$ \\
H & 8 & 15 & $(09 / 10 ; 90,0 \%)$ \\
I & 9 & 10 & $(14 / 20 ; 70,0 \%)$ \\
\hline J & 13 & 20 & $(17 / 33 ; 51,5 \%)$ \\
\hline K & 27 & 33 & \\
\hline
\end{tabular}

Legenda: taxa de prenhez; dentro do parêntese, número de fêmeas inseminadas por números de fêmeas com prenhez mantida aos 60 dias de gestação. Fonte: autores.

\section{Discussão}

Observou-se que a motilidade subjetiva a campo foi superior à análise de motilidade realizada pelo método automatizado (CASA). É importante ressaltar que na análise subjetiva, o nível de correlação pode sofrer u variação entre 0,15 e 0,83 (Januskauskas et al., 2003) e a estimativa pela análise computadorizada, entre 0,82 e 0,98 (Farrell et al., 1996). Segundo Carvalho et al. (2009), durante a avaliação subjetiva, há uma tendência em superestimar a percentagem de espermatozoides móveis, especialmente em amostras com alta concentração espermática. Esta superestimação, entretanto, não é comum para a espécie equina que apresenta ejaculado com maior proporção de plasma seminal em relação aos espermatozoides.

Deve-se considerar que as amostras foram acondicionadas, refrigeradas e transportadas para análise em outra localidade, o que acrescentou, em média, três horas de transporte para a avaliação. Sieme et al. (2015) afirmaram que espermatozoides são mais resistentes a refrigeração quando possuem altas concentrações de colesterol na membrana plasmática, que atua estabilizando-a e reduzindo a faixa de temperatura para a fase de transição. As avaliações realizadas neste estudo mostraram que, em condições de campo, a célula espermática tende a possuir maior motilidade, entretanto, pósrefrigeração, há redução da motilidade, como observado na avaliação fornecida pelo método CASA, mesmo que em um curto período de tempo.

Ao avaliar a integridade e funcionalidade de membrana espermática, observou-se correlação positiva $(0,69)$ entre o teste de dupla coloração com eosina/nigrosina e a sonda fluorófora IPD. Pintado et al. (2000) ratificaram o papel do IPD como um bom indicador de células lesadas, pois observaram correlação positiva com a eosina/nigrosina/giemsa. Esta observação, no presente experimento, reforça que o teste eosina/nigrosina pode ser utilizado de forma segura a campo, pois os resultados obtidos são compatíveis à técnica de citometria de fluxo, sendo o primeiro um teste mais barato e prático de execução a campo para exame clínico andrológico em equinos.

Vale ressaltar que a idade interferiu negativamente sobre a manutenção da motilidade. Roser (2001) cita a hipótese de garanhões com idade acima de quinze anos apresentarem redução na fertilidade, condição associada à redução na produção de 
espermatozoides com motilidade progressiva e possível degeneração testicular; estas falhas reprodutivas iniciam-se precocemente, em nível testicular, evoluindo para o sistema endócrino.

A redução da qualidade seminal ou da fertilidade em animais mais velhos é perceptível neste experimento, que não evidenciou diferença nos parâmetros clínicos dos equinos avaliados, porém estas alterações foram visualizadas no exame ultrassonográfico e nos resultados de prenhez. Arruda et al. (2010) orientam que a nutrição dos animais idosos é fundamental para a obtenção de bons resultados na reprodução e alterações neste aspecto poderiam comprometer a fertilidade de garanhões. Nas condições deste experimento podemos descartar a questão alimentar, visto que os animais receberam alimentação balanceada de acordo com a idade, assim como foi verificada a capacidade de ingestão de alimento por estes animais, sendo descartados alterações dentárias ou digestórias. Desta forma, é interessante abordar possíveis falhas reprodutivas.

Pavlica e Barozz (2001) citaram que a funcionalidade do testículo está associada a uma boa perfusão testicular. Esta afirmação justifica os resultados encontrados neste experimento em animais a partir de seis anos de idade, uma vez que os animais mais velhos apresentaram o índice de resistividade menor em comparação aos animais mais jovens, dados corroborados por Ortega-Ferrusola et al. (2014). Schurich et al. (2009) relataram que o índice de resistência (IR) tem relação direta com a perfusão normal nos tecidos; qualquer alteração tissular nos testículos acarreta em alterações nas células espermáticas, já que a massa testicular é constituída entre 70 a $80 \%$ de túbulos seminíferos, e esses possuem uma concentração de oxigênio relativamente baixa quando comparado com outros órgãos. Menor resistividade indica maior fluxo sanguíneo, que eleva a temperatura testicular e pode culminar em degeneração testicular, prejudicando e reduzindo a espermatogênese (Alvarenga \& Papa, 2009).

Neste estudo, com o aumento da idade do garanhão foi percebido menor resistividade vascular, e pode indicar interferência na espermatogênese e consequente menor qualidade espermática. Nos resultados obtidos neste experimento foi verificada correlação positiva entre IP e ALH. De acordo com Verstegen et al. (2002), a ALH é um parâmetro indicativo de fertilidade devido à capacidade de penetração da célula espermática na zona pelúcida do oócito. Entretanto, o aumento da ALH, ainda no momento da avaliação seminal, é classificada como uma capacitação espermática precoce e pode justificar a diminuição da fertilidade de reprodutores mais velhos, considerando ainda a inseminação com sêmen refrigerado, uma vez que o sêmen apresentaria menor tempo de viabilidade no trato reprodutor da fêmea e comumente a inseminação não ocorreria no momento da ovulação, ainda mais na espécie equina, que apresenta uma janela de ovulação (Pimentel et al., 2014).

O exame clínico andrológico, pela avaliação dos parâmetros de motilidade subjetiva, integridade e funcionalidade de membrana plasmática não justificariam a diminuição da fertilidade em animais mais velhos, assim como a alteração nos parâmetros cinéticos, entretanto, a adição do exame ultrassonográfico com a ferramenta doppler pôde fundamentar tais achados.

\section{Conclusão}

Conclui-se que o exame clínico andrológico, quando isolado, é limitado para atestar a capacidade fértil de garanhões; a ultrassonografia associada ao método doppler identifica animais subférteis e pode ser uma ferramenta auxiliar ao exame clínico andrológico na seleção de reprodutores equinos.

\section{Agradecimentos}

O presente trabalho foi realizado com apoio da Fundação de Apoio à Pesquisa do estado da Paraíba (FAPESQ-PB) e da Coordenação de Aperfeiçoamento de Pessoal de Nível Superior/Brasil (CAPES) - Código de Financiamento 001. 


\section{Referências}

Agência Executiva de Gestão das Águas do Estado da Paraíba - AESA. (2017). Climatologia. http://www.aesa.pb.gov.br/aesawebsite/meteorologiachuvas/climatologia.

Alvarenga, M. A., \& Papa, F. O. (2009) Principais distúrbios reprodutivos observados em garanhões no Brasil. Revista Brasileira de Reprodução Animal, 6, 204-209. http://www.cbra.org.br/pages/publicacoes/rbra/download/p204-209.pdf

Arruda, R. P., Silva, D. F., Alonso, M. A., Andrade, A. F. C.; Nascimento, J., Gallego, A. M., Martins, S. M. M. K., \& Granato, T. M. (2010) Nutraceuticals in reproduction of bulls and stallions. Revista Brasileira de Zootecnia, 39, 393-400. https://doi.org/10.1590/S1516-35982010001300043

Carvalho, J.O.; Sartori, R.; Lemes, A.P., Mourão, G. B., \& Dode, M A. N. (2009) Cinética de espermatozoides criopreservados de bovinos após sexagem por citometria de fluxo. Pesquisa Agropecuária Brasileira, 44(10), 1346-1351. https://doi.org/10.1590/S0100-204X2009001000019

CBRA - Colégio Brasileiro de Reprodução Animal (2013). Manual para Exame Andrológico e Avaliação de Sêmen Animal. Third ed. CBRA, Belo Horizonte. 46p.

Coelho, R. W. de A., \& Dias, J. C. O. (2021). Congelação de sêmen equino após 24 horas de resfriamento. Research, Society and Development, 10(1), e1831019939. https://doi.org/10.33448/rsd-v10i1.9939

Cunha, M. S., Bonato, D. V., Taira, A. R., Teixeira, P. P. M. Degeneração testicular em machos: dos animais ao homem. Investigação, 14(6):54-61, 2015. https://doi.org/10.26843/investigacao.v14i6.912.

Farrell, P. B., Foote, R. H., McArdle, M. M., Trouern-Trend, V. L., \& Tardif, A. L. (1996). Media and dilution procedures tested to minimize handling effects on human, rabbit, and bull sperm for computer-assisted sperm analysis (CASA). Journal of Andrology, 17(3), 293-300.

Ginther, O. J. (1992). Reproductive biology of the mare (Basic and applied aspects). 2nd ed. Cross Plains: Equiservices, 642p.

Hammes, A. M.; Pimentel, C. A.; Fernandes, C. E. (1996). Fertilidades em garanhões avaliadas através do exame andrológico. Ciência Rural, 26, 277-283. https://www.scielo.br/j/cr/a/CQmcSRnR3qY3Rv7w53qynbP/?format=pdf\&lang=pt

Januskauskas, A., Johannisson, A., \& Rodriguez-Martinez, H. (2003). Subtle membrane changes in cryopreserved bull semen in relation with sperm viability, chromatin structure, and field fertility. Theriogenology, 60(4), 743-758. https://doi.org/10.1016/s0093-691x(03)00050-5

Linhares, R. S., \& Sousa, M. S. de. (2020). Uma perspectiva jurídica sobre a relevância socioeconômica e cultural da vaquejada e seus reflexos no direito dos animais. Revista do programa de pós-graduação em gestão ambiental no semiárido, 2(1), 01-20. https://www.gvaa.com.br/revista/index.php/Regeas/article/view/7984

Nery, I., Araújo Silva, R., Souza, H. M., Arruda, L., Monteiro, M. M., Seal, D., Silva, G. R., Silva, T., Carneiro, G. F., Batista, A. M., Câmara, D. R., \& Guerra, M. (2020). Effects of L-Carnitine on Equine Semen Quality During Liquid Storage. Biopreservation and Biobanking, 18(5), 403-408. https://doi.org/10.1089/bio.2020.0025

Obino Filho, F. (2016) Palavra do Presidente. In: Lima, R.A.S.; \& Cintra, A.G. Revisão do Estudo do Complexo do Agronegócio do Cavalo. Assessoria de Comunicação e Eventos do Ministério da Agricultura, Pecuária e Abastecimento. https://www.gov.br/agricultura/pt-br/assuntos/camaras-setoriaistematicas/documentos/camaras-setoriais/equideocultura/anos-anteriores/revisao-do-estudo-do-complexo-do-agronegocio-do-cavalo/view.

Ortega-Ferrusola, C., Gracia-Calvo, L. A., Ezquerra, J., \& Pena, F. J. (2014). Use of colour and spectral Doppler ultrasonography in stallion andrology. Reproduction in domestic animals = Zuchthygiene, 49 Suppl 4, 88-96. https://doi.org/10.1111/rda.12363

Papa, F. O., Alvarenga, M. A., Dell'qua, J.; Monteiro, G. M. (2007) Manual de andrologia e manipulação de sêmen equino. São Paulo: Botupharma, 60p.

Pavlica, P., \& Barozzi, L. (2001). Imaging of the acute scrotum. European Radiology, 11(2), 220-228. https://doi.org/10.1007/s003300000604

Pereira, E. L., Bataglion, G. A., \& Mazo, J. Z. (2020) Equoterapia, saúde e esporte: figurações da prática no Rio Grande do Sul, 1970-2000. História, Ciências, Saúde-Manguinhos, 27(3) 879-897. https://doi.org/10.1590/S0104-59702020000400010.

Pimentel, M. M. L., Santos, F. A. Dos, Dias, R. V. C., Macêdo, L. B. De, Fonseca, Z. A. A. De S., André, W. P. P., \& Ribeiro, W. L. C. (2014). Monitoramento do ciclo estral de fêmeas equinas por meio de citologia vaginal, ultrassonografia e dosagem hormonal. Arquivos de Ciências Veterinárias e Zoologia da UNIPAR, 17(1), 69-75. https://doi.org/10.25110/arqvet.v17i1.4920

Pintado, B., de la Fuente, J., \& Roldan, E. R. (2000). Permeability of boar and bull spermatozoa to the nucleic acid stains propidium iodide or Hoechst 33258 , or to eosin: accuracy in the assessment of cell viability. Journal of Reproduction and Fertility, 118(1), 145-152. https://pubmed.ncbi.nlm.nih.gov/10793636/

Roser J. F. (2001). Endocrine and paracrine control of sperm production in stallions. Animal Reproduction Science, 68(3-4), 139-151. https://doi.org/10.1016/s0378-4320(01)00151-8

Schurich, M., Aigner, F., Frauscher, F., \& Pallwein, L. (2009). The role of ultrasound in assessment of male fertility. European Journal of Obstetrics, Gynecology, and Reproductive Biology, 144 Suppl 1, S192-S198. https://doi.org/10.1016/j.ejogrb.2009.02.034

Sieme, H., Oldenhof, H., \& Wolkers, W. F. (2015). Sperm Membrane Behaviour during Cooling and Cryopreservation. Reproduction in domestic animals = Zuchthygiene, 50 Suppl 3, 20-26. https://doi.org/10.1111/rda.12594

Smith, A. M.; Staniar, W. B.; \& Splan, R. K. Associations between yearling body measurements and career racing performance in Thoroughbred racehorses. Journal of Equine Veterinary Science, 26(5), 212-214. https://doi.org/10.1016/j.jevs.2006.03.002 
Research, Society and Development, v. 10, n. 14, e582101422412, 2021

(CC BY 4.0) | ISSN 2525-3409 | DOI: http://dx.doi.org/10.33448/rsd-v10i14.22412

Snoeck, P. P. d. N., Melo, M. I. V. d., Alves, S. G. G., Bittencourt, R. F., Filho, A. d. L. R., Chalhoub, M., \& Henry, M. (2014). Qual é o teste hiposmótico mais indicado para avaliar a integridade funcional de espermatozoides equino criopreservados? Brazilian Journal of Veterinary Medicine, 36(4), 335-361. http://rbmv.org/index.php/BJVM/article/view/562

Verstegen, J., Iguer-Ouada, M., \& Onclin, K. (2002). Computer assisted semen analyzers in andrology research and veterinary practice. Theriogenology, 57(1), 149-179. https://doi.org/10.1016/s0093-691x(01)00664-1 